\title{
March 2002
}

\section{Numerical evaluation of the general massive 2-loop sunrise self-mass master integrals from differential equations.}

\author{
M. Caffo ${ }^{a b}$, H. Czyż ${ }^{c}$ and E. Remiddi ${ }^{b a}$ \\ a INFN, Sezione di Bologna, I-40126 Bologna, Italy \\ ${ }^{b}$ Dipartimento di Fisica, Università di Bologna, I-40126 Bologna, Italy \\ ${ }^{c}$ Institute of Physics, University of Silesia, PL-40007 Katowice, Poland \\ e-mail: caffo@bo.infn.it \\ czyz@us.edu.pl \\ remiddi@bo.infn.it
}

\begin{abstract}
The system of 4 differential equations in the external invariant satisfied by the 4 master integrals of the general massive 2-loop sunrise self-mass diagram is solved by the Runge-Kutta method in the complex plane. The method, whose features are discussed in details, offers a reliable and robust approach to the direct and precise numerical evaluation of Feynman graph integrals.
\end{abstract}

PACS 11.10.-z Field theory

PACS 11.10.Kk Field theories in dimensions other than four

PACS 11.15.Bt General properties of perturbation theory

PACS 12.20.Ds Specific calculations PACS 12.38.Bx Perturbative calculations

*Work supported in part by the European Community's Human Potential Programme under contract HPRN-CT-2000-00149 Physics at Colliders. 


\section{Introduction.}

High precision measurements in high energy physics (or more in general in the determination of particle properties) require more and more precise calculations of multi-loop Feynman diagrams to have sufficiently precise theoretical predictions to compare with.

The nowadays widely accepted procedure of expressing radiative correction calculations in terms of a limited number of master integrals (MI) [1] reduces the problem to the careful determination of these quantities. The method has also the advantage that, with a correct bookkeeping of the recurrence relations arising from integration by parts identities, the MI of a given problem can be reused in more complicated calculations.

The analytical calculation of MI, in terms of the usual polylogarithms and their generalizations, is in general possible only when the number of different scales (internal masses and external momenta or Mandelstam variables) is small, like in QCD calculations, where all masses are set to zero or in the QED cases, where only the electron mass is different from zero, or when the external variables are fixed to particular values (zero or mass shell condition). Another possibility of big help in analytic calculations is sometimes offered by the exploitation of particular simplifying conditions, like the smallness of some ratios of the parameters allowing the corresponding expansion.

In the general massive case, relevant in the electroweak theory, the number of parameters prevents from obtaining results in the usual analytic form already in the case of the 2-loop sunrise self-mass diagram shown in Fig.1.

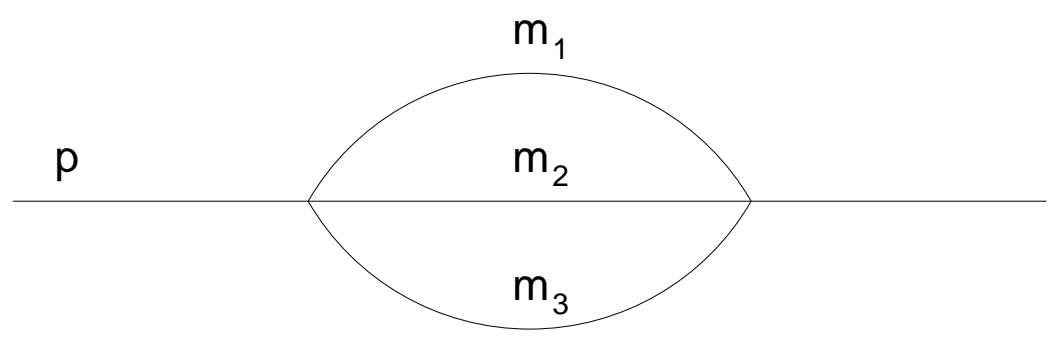

Figure 1: The general massive 2-loop sunrise self-mass diagram.

This diagram has indeed a long history of investigation and its MI were even recognized to be expressible in closed form as a combination of four Lauricella functions, a special class of generalized hypergeometric series [2] (and earlier references therein). 
The method provides efficient multiple series expansions for the regions of small $\left|p^{2}\right|$, i.e. $\left|p^{2}\right|<\max \left(m_{i}^{2}\right)$, and of large $\left|p^{2}\right|$, i.e. $\left|p^{2}\right|>\left(m_{1}+m_{2}+m_{3}\right)^{2}$, but some problems arise in the intermediate region.

Great efforts were therefore devoted to investigate the properties in the special points (i.e. $p^{2}=0, \infty$, pseudothresholds and threshold). The analytical expansions of the MI at 0 and $\infty$ are given in [2] and [3]; the values at pseudothresholds and threshold in [四]; the analytical expansions at pseudothresholds are in [5]; a semi-analytical expansion at threshold is in [6] and also in configuration space technique in [0], while the complete analytical expansions at threshold are presented in [8].

For numerical evaluation purposes, it is possible to cast the general massive self-mass diagram as a double integral representation and in the particular case of the sunrise diagram in a single integral representation [2], [9] (and earlier references therein). The configuration space technique is also exploited in the numerical approach [2], [10]. In a recent approach rearrangements of the integrand, driven by the Bernstein-Tkachov theorem, are introduced to improve numerical convergence [11]. A different and interesting method is the use of the recurrence relations as difference equations to numerically evaluate the MI [12].

In the present paper we exploit the numerical evaluation of the four MI related to the general massive 2-loop sunrise self-mass diagram [13], using the differential equations in $p^{2}$, obtained in [3] and the Runge-Kutta method [14] to solve them for complex values of $p^{2}$. The interest is not limited to provide with a fast routine precise numerical values for all the four MI in the general massive case and for all the values of $p^{2}$, but extends to the investigation of the reliability of the method, as it can be easily extended to the numerical evaluation of other less studied diagrams.

In Section 2 the master differential equations are recalled and the analytic properties of the MI are reviewed. Section 3 contains a description of the method used to solve the system of differential equations and to determine the accuracy. In Section 4 the control tests and the comparisons with other values reported in the literature are presented. Finally in Section 5 our conclusions on the application of the method to present and further work are presented. 


\section{Analytical properties and behaviours of the MI.}

We use here the following definition of the four MI related to the general massive 2-loop sunrise self-mass diagram in $n$ continuous dimensions and with fully Euclidean variables

$$
\begin{aligned}
& F_{j}\left(n, m_{1}^{2}, m_{2}^{2}, m_{3}^{2}, p^{2}\right)=\frac{\mu^{8-2 n}}{\left((2 \pi)^{n-2}\right)^{2}} \\
& \left.\quad \int d^{n} k_{1} \int d^{n} k_{2} \frac{1}{\left(k_{1}^{2}+m_{1}^{2}\right)^{\alpha_{1}(j)}\left(k_{2}^{2}+m_{2}^{2}\right)^{\alpha_{2}(j)}\left(\left(p-k_{1}-k_{2}\right)^{2}+m_{3}^{2}\right)^{\alpha_{3}(j)}}, j=0,1,2, ß 1\right)
\end{aligned}
$$

and $i=1,2,3 ; \alpha_{i}(0)=1$, for $j=0 ; \alpha_{i}(j)=1$, for $j \neq i ; \alpha_{i}(j)=2$, for $j=i$.

Wherever necessary to avoid ambiguities, the usual imaginary displacements $m_{i}^{2} \rightarrow$ $m_{i}^{2}-i \epsilon$, where $\epsilon$ is an infinitesimal positive number, are understood.

At variance from [3], were the mass scale was given the value $\mu=1$, here we choose

$$
\mu=m_{1}+m_{2}+m_{3}
$$

which comes out to be the appropriate mass scale parameter for the numerical discussion. The expansion of the MI around $n=4$ has the form [3]

$$
\begin{aligned}
F_{j}\left(n, m_{1}^{2}, m_{2}^{2}, m_{3}^{2}, p^{2}\right)=C^{2}(n)\left\{\frac{1}{(n-4)^{2}} F_{j}^{(-2)}\left(m_{1}^{2}, m_{2}^{2}, m_{3}^{2}, p^{2}\right)\right. \\
\left.+\frac{1}{(n-4)} F_{j}^{(-1)}\left(m_{1}^{2}, m_{2}^{2}, m_{3}^{2}, p^{2}\right)+F_{j}^{(0)}\left(m_{1}^{2}, m_{2}^{2}, m_{3}^{2}, p^{2}\right)+\mathcal{O}(n-4)\right\} .
\end{aligned}
$$

where the coefficient $C(n)$

$$
C(n)=(2 \sqrt{\pi})^{(4-n)} \Gamma\left(3-\frac{n}{2}\right),
$$

not expanded, can be replaced by its value $C(4)=1$, at $n=4$, when multiplying a function regular in $(n-4)$. The coefficients of the poles in $(n-4)$ of $F_{0}\left(n, m_{1}^{2}, m_{2}^{2}, m_{3}^{2}, p^{2}\right)$ are known to be [3]

$$
\begin{aligned}
F_{0}^{(-2)}\left(m_{1}^{2}, m_{2}^{2}, m_{3}^{2}, p^{2}\right)= & -\frac{1}{8}\left(m_{1}^{2}+m_{2}^{2}+m_{3}^{2}\right) \\
F_{0}^{(-1)}\left(m_{1}^{2}, m_{2}^{2}, m_{3}^{2}, p^{2}\right)= & \frac{1}{8}\left\{\frac{p^{2}}{4}+\frac{3}{2}\left(m_{1}^{2}+m_{2}^{2}+m_{3}^{2}\right)\right. \\
& \left.-\left[m_{1}^{2} \log \left(\frac{m_{1}^{2}}{\mu^{2}}\right)+m_{2}^{2} \log \left(\frac{m_{2}^{2}}{\mu^{2}}\right)+m_{3}^{2} \log \left(\frac{m_{3}^{2}}{\mu^{2}}\right)\right]\right\}
\end{aligned}
$$


while those for $F_{i}\left(n, m_{1}^{2}, m_{2}^{2}, m_{3}^{2}, p^{2}\right), i=1,2,3$ are

$$
\begin{aligned}
& F_{i}^{(-2)}\left(m_{1}^{2}, m_{2}^{2}, m_{3}^{2}, p^{2}\right)=\frac{1}{8} \\
& F_{i}^{(-1)}\left(m_{1}^{2}, m_{2}^{2}, m_{3}^{2}, p^{2}\right)=-\frac{1}{16}+\frac{1}{8} \log \left(\frac{m_{i}^{2}}{\mu^{2}}\right)
\end{aligned}
$$

From now on we deal only with the finite parts of the MI (i.e. we subtract the $n-4$ poles) and we do not write anymore, for short, the arguments of the functions $F_{j}^{(0)}\left(m_{1}^{2}, m_{2}^{2}, m_{3}^{2}, p^{2}\right) \equiv F_{j}^{(0)}$, unless we need to refer explicitly to them.

The differential equations satisfied by the finite part of the MI expansions, given in [3], can be written as

$$
\begin{array}{r}
p^{2} \frac{\partial}{\partial p^{2}} F_{0}^{(0)}=F_{0}^{(0)}+m_{1}^{2} F_{1}^{(0)}+m_{2}^{2} F_{2}^{(0)}+m_{3}^{2} F_{3}^{(0)}+T_{0} \\
p^{2} D\left(m_{1}^{2}, m_{2}^{2}, m_{3}^{2}, p^{2}\right) \frac{\partial}{\partial p^{2}} F_{i}^{(0)}=\sum_{j=0}^{3} M_{i, j} F_{j}^{(0)}+T_{i} \quad, i=1,2,3
\end{array}
$$

where the explicit form of the functions $T_{0}, T_{i}$ (polynomials of $p^{2}$ and $m_{i}^{2}$, and logarithms of $m_{i}^{2} / \mu^{2}$ ) and $M_{i, j}$ (polynomials of $p^{2}$ and $m_{i}^{2}$ ) can be found in [3]. The function $D\left(m_{1}^{2}, m_{2}^{2}, m_{3}^{2}, p^{2}\right)$ is defined by

$$
\begin{aligned}
D\left(m_{1}^{2}, m_{2}^{2}, m_{3}^{2}, p^{2}\right) & =\left(p^{2}-p_{t h}^{2}\right)\left(p^{2}-p_{p s 1}^{2}\right)\left(p^{2}-p_{p s 2}^{2}\right)\left(p^{2}-p_{p s 3}^{2}\right) \\
p_{t h}^{2} & =-\left(m_{1}+m_{2}+m_{3}\right)^{2} \\
p_{p s 1}^{2} & =-\left(m_{1}+m_{2}-m_{3}\right)^{2} \\
p_{p s 2}^{2} & =-\left(m_{1}-m_{2}+m_{3}\right)^{2} \\
p_{p s 3}^{2} & =-\left(m_{1}-m_{2}-m_{3}\right)^{2}
\end{aligned}
$$

and vanishes at the threshold $p_{t h}^{2}$ and at the three pseudothresholds $p_{p s 1}^{2}, p_{p s 2}^{2}, p_{p s 3}^{2}$. Indeed the values of $p^{2}$ at which the coefficients of the derivatives in Eq.(17) vanish, together with $p^{2}=\infty$, are the singular points of the differential equations, which we will call special points because special care is required in the numerical computation of the MI.

The differential equations Eq.([7) allow a class of solutions wider than just the functions Eq.(1), but the initial conditions at $p^{2}=0$ imposed by Eq.(11) identify uniquely the solutions (actually the regularity at $p^{2}=0$, even without the explicit knowledge of the functions in that point, is enough to fix the solutions). Once the initial condition at $p^{2}=0$ is fixed, one finds that the pseudo-threshold values $p^{2}=p_{p s 1}^{2}, p^{2}=p_{p s 2}^{2}, p^{2}=p_{p s 3}^{2}$ are also 
regular points [5], while the threshold value $p^{2}=p_{t h}^{2}$ is a branch point [8] (in agreement, of course, with standard textbook results [15]).

It is convenient to use reduced masses and reduced external invariant

$$
m_{i, r} \equiv \frac{m_{i}}{m_{1}+m_{2}+m_{3}} \quad, \quad p_{r}^{2} \equiv \frac{p^{2}}{\left(m_{1}+m_{2}+m_{3}\right)^{2}},
$$

together with a dimensionless version of $F_{0}^{(0)}$, defined by

$$
F_{0, r}^{(0)} \equiv \frac{F_{0}^{(0)}}{\left(m_{1}+m_{2}+m_{3}\right)^{2}}
$$

as the other master integrals are already dimensionless, the values of all the functions are now pure numbers. In terms of the new variables $p_{r}^{2}, m_{i, r}$ the threshold is located at $p_{t h, r}^{2}=p_{t h}\left(m_{i, r}\right)=-1$ and the pseudo-thresholds are in $p_{p s 1, r}^{2}=p_{p s 1}\left(m_{i, r}\right), p_{p s 2, r}^{2}=$ $p_{p s 2}\left(m_{i, r}\right), p_{p s 3, r}^{2}=p_{p s 3}\left(m_{i, r}\right)$. We also recall here that according to the Euclidean definition our $p^{2}$ is positive for space-like $p$, and negative for time-like $p$.

Typical plots of the real and imaginary parts of the MI for two sets of masses are shown in Figures 2, 3, A and 5. The plots are obtained by means of the FORTRAN program described in the next two sections and each consists of 6000 points per function calculated with the relative precision of $10^{-6}$.

The behavior of the functions depends of course on the values of the masses, however some of their properties are quite general, as it appears from the following discussion. The real part of $F_{0, r}^{(0)}$ as seen in figures 2 and 3 has one local minimum and one local maximum for finite $p_{r}^{2}$ and goes to $\pm \infty$ for $p_{r}^{2} \rightarrow \pm \infty$. We do not have analytically the exact position of the local extrema, but as they appear to lie outside the region $-1 \leq p_{r}^{2} \leq 1$ they can be found approximately with the help of the asymptotic expansion (large $p_{r}^{2}$ ) for $F_{0, r}^{(0)}$, which is [3]

$$
F_{0, r}^{(0)}=\frac{p_{r}^{2}}{32}\left(\log \left(p_{r}^{2}\right)-\frac{13}{4}\right)+\frac{1}{32} \log ^{2}\left(p_{r}^{2}\right) \sum_{i=1}^{3} m_{i, r}^{2}-\frac{1}{16} \log \left(p_{r}^{2}\right) \sum_{i=1}^{3} m_{i, r}^{2} \log \left(m_{i, r}^{2}\right)+\cdots
$$

The asymptotic behavior of $\operatorname{Re} F_{0, r}^{(0)}$ is obvious from Eq.(11), while for the positions of the maximum and minimum one finds approximately from the first term only, which is also the leading, $p_{r, \max }^{2}=-9.5$ and $p_{r, \text { min }}^{2}=9.5$, independent from the mass values. The approximate values of the function at those points, again from the first term only, are $\operatorname{Re} F_{0, r}^{(0)}\left(p_{r, \max }^{2}\right)=0.3$ and $\operatorname{Re} F_{0, r}^{(0)}\left(p_{r, \min }^{2}\right)=-0.3$, also independent from the mass values. Taking into account the asymptotic behavior one expects at least three zeros of 


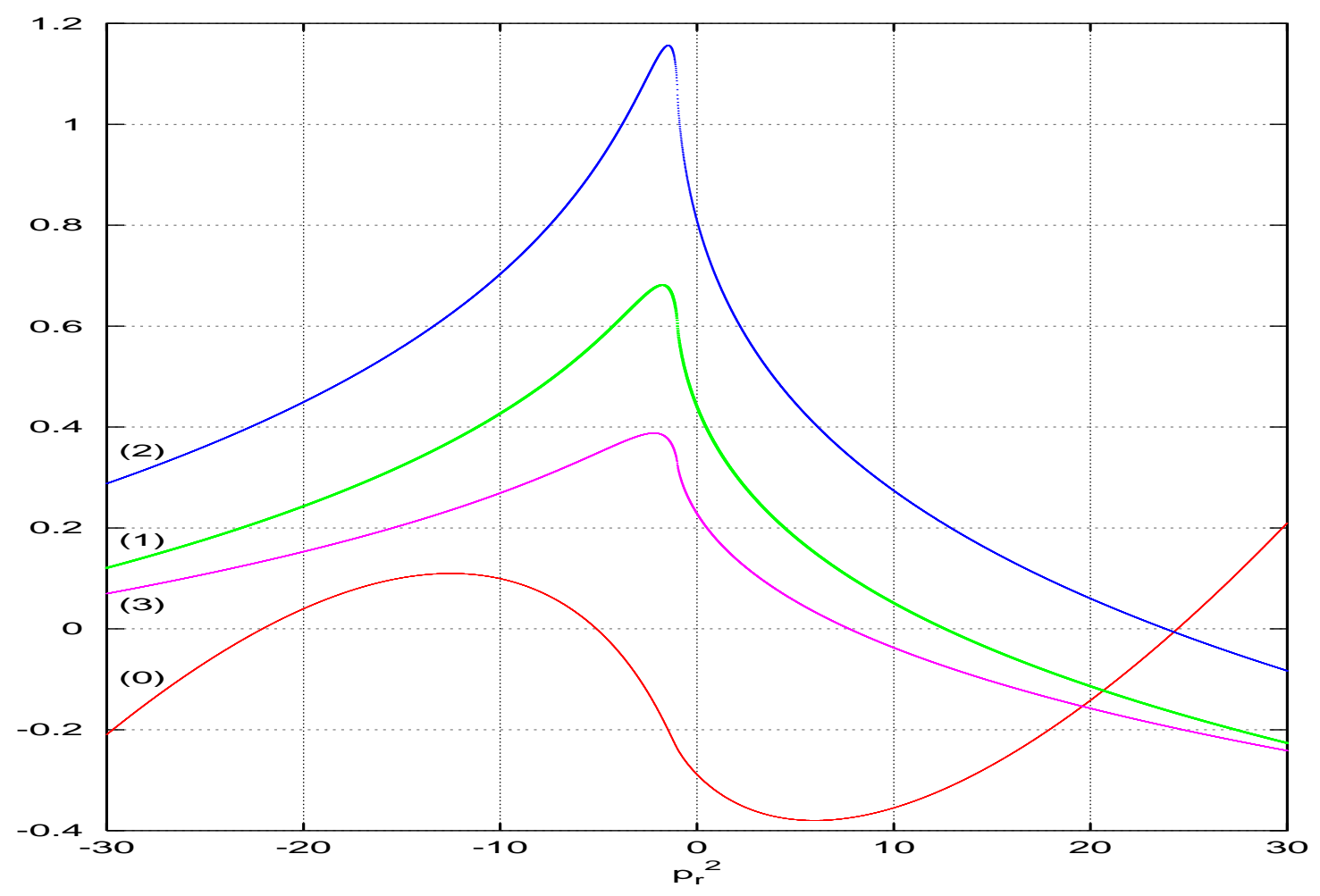

Figure 2: Plots of Re $F_{0, r}^{(0)}$ (labeled as $\left.(0)\right)$ and $\operatorname{Re} F_{i}^{(0)}$ (labeled as $(i)$ ) as a function of $p_{r}^{2}$ for $m_{1}=2, m_{2}=1, m_{3}=4$ and $\mu=m_{1}+m_{2}+m_{3}$.

the function, provided that in non asymptotic region there are no additional extrema (which is actually the case). The second derivative of $\operatorname{Re} F_{0, r}^{(0)}$ at threshold is infinite [8], but it does not change sign at that point, even if the position of the flex point of $\operatorname{Re} F_{0, r}^{(0)}$ is not far from the threshold.

The other MI go one into the other by the exchange of the values of the related masses. From the expression of their expansion for large $p_{r}^{2}$

$$
F_{i}^{(0)}=-\frac{1}{32} \log ^{2}\left(p_{r}^{2}\right)+\frac{1}{16} \log \left(p_{r}^{2}\right)\left(\log \left(m_{i, r}^{2}\right)+1\right)+\cdots,
$$

we see that their real parts all go to $-\infty$ in both asymptotic regions $p_{r}^{2} \rightarrow \pm \infty$, however the position of the maximum cannot be obtained just from the first terms of the asymptotic expansion, as it is positioned in the region of small $p_{r}^{2}$. The analytic expansions at threshold [8] show that the derivatives of the Re $F_{i}^{(0)}$ are infinite, but they do not change 


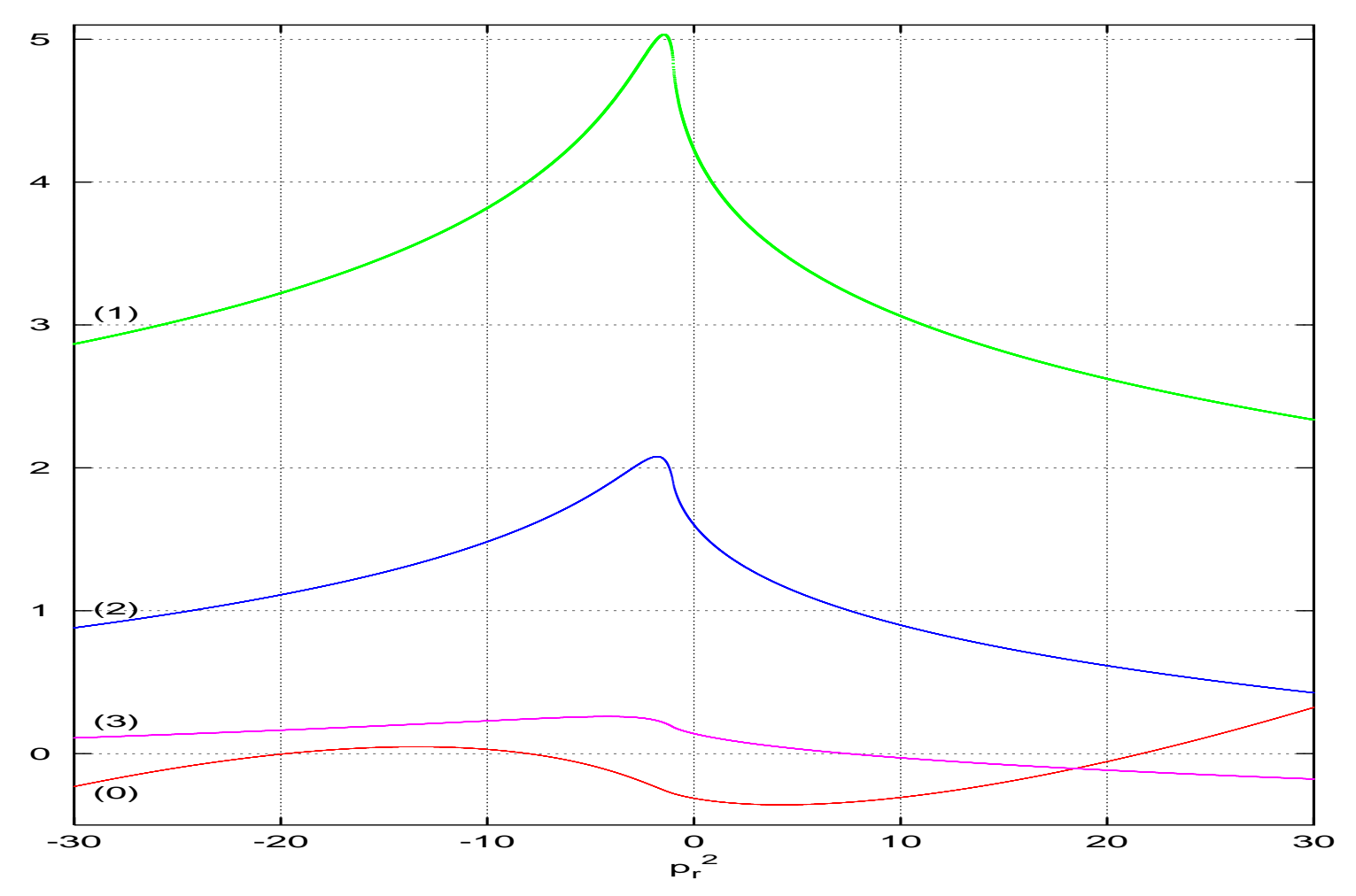

Figure 3: Plots of Re $F_{0, r}^{(0)}$ (labeled as (0)) and Re $F_{i}^{(0)}$ (labeled as $(i)$ ) as a function of $p_{r}^{2}$ for $m_{1}=1, m_{2}=9, m_{3}=200$ and $\mu=m_{1}+m_{2}+m_{3}$.

sign exactly at that point.

The imaginary parts of all the functions plotted in figures 1 and 5 exhibit no complicated structure and their asymptotic behaviors can be simply deduced from Eq.(11) and Eq.(12). Observe that, due to our definition of $p_{r}^{2}$, the proper analytic continuation for time-like $p$, hence negative $p_{r}^{2}=-\left|p_{r}^{2}\right|$, is obtained by giving a positive infinitesimal imaginary part to $-p_{r}^{2}$, so that $\log \left(p_{r}^{2}\right) \rightarrow \log \left(p_{r}^{2}-i \epsilon\right)=\log \left|p_{r}^{2}\right|-i \pi$ (at variance with the default option of FORTRAN compilers).

\section{The numerical method.}

For the numerical solution of the system of differential equations we use fourth-order Runge-Kutta method [14]. The method starts from the known values of the solutions in 


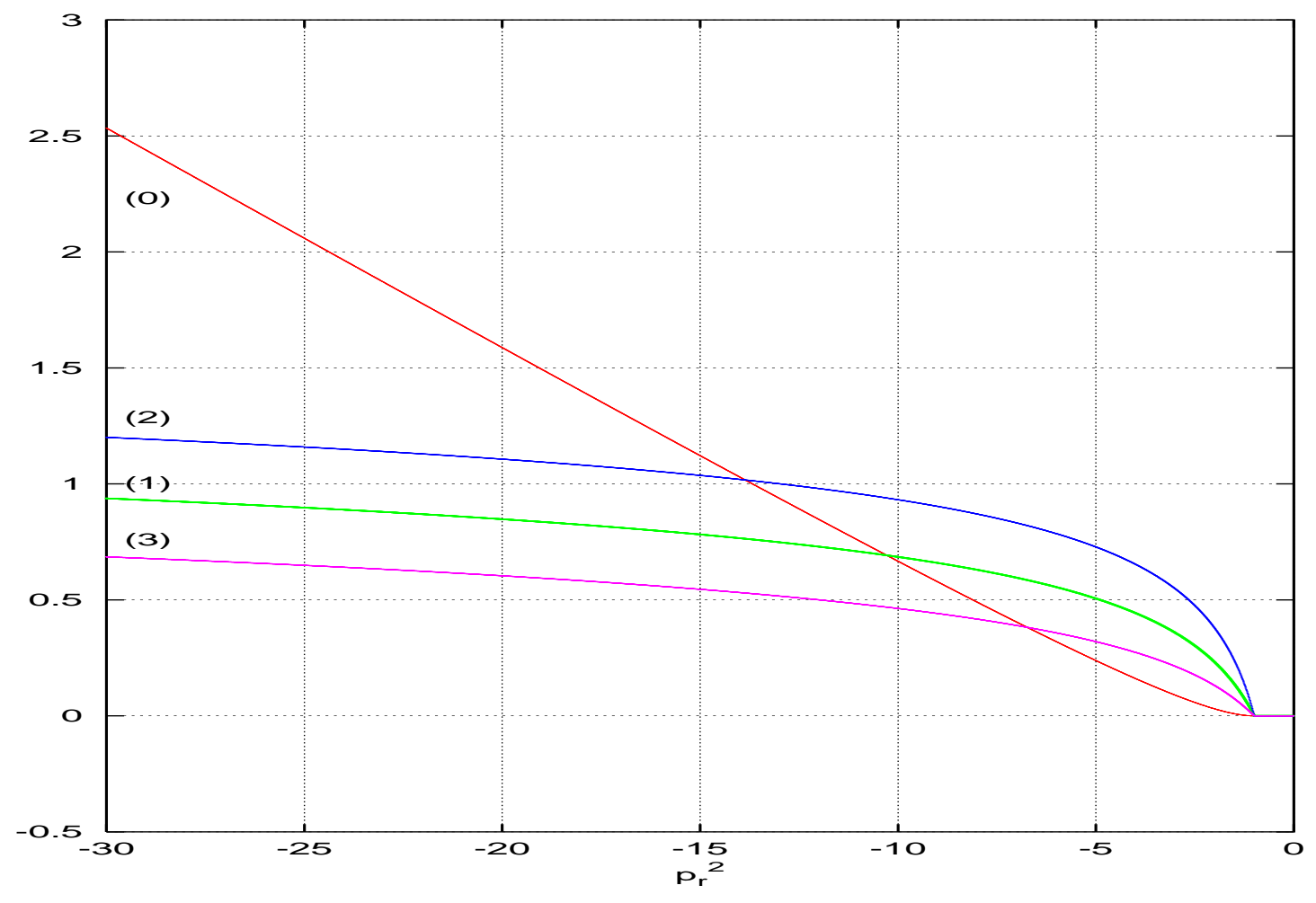

Figure 4: Plots of $\operatorname{Im} F_{0, r}^{(0)}$ (labeled as $\left.(0)\right)$ and $\operatorname{Im} F_{i}^{(0)}$ (labeled as $(i)$ ) as a function of $p_{r}^{2}$ for $m_{1}=2, m_{2}=1, m_{3}=4$ and $\mu=m_{1}+m_{2}+m_{3}$.

an initial point, then it calculates the values of the solutions in a nearby point at distance $\Delta$ with an expansion in $\Delta$ based on the differential equations, omitting terms of order $\Delta^{5}$. Repeating the procedure along a path of length $L$ in $N$ steps, so that the step is of length $\Delta=L / N$, a relative error of approximately $N \Delta^{5}=L^{5} / N^{4}$ is accumulated, and the requested accuracy is obtained by a suitable choice of $L$ and $N$. This method is known for its robustness, and indeed it works quite well in our case allowing us to obtain a relative accuracy of $10^{-10}-10^{-12}$ (the FORTRAN program is written in double precision) within reasonable CPU time (see discussion at end of this section). More sophisticated methods exist and could be implemented, but the simplicity of the used one has the advantage of a better control on the accuracy.

To obtain the four MI related to the general massive 2-loop sunrise self-mass diagram we use the system of four linear differential equations given in Eq.(7). For the necessary initial conditions we use the values of the MI in the special points, where the differential 


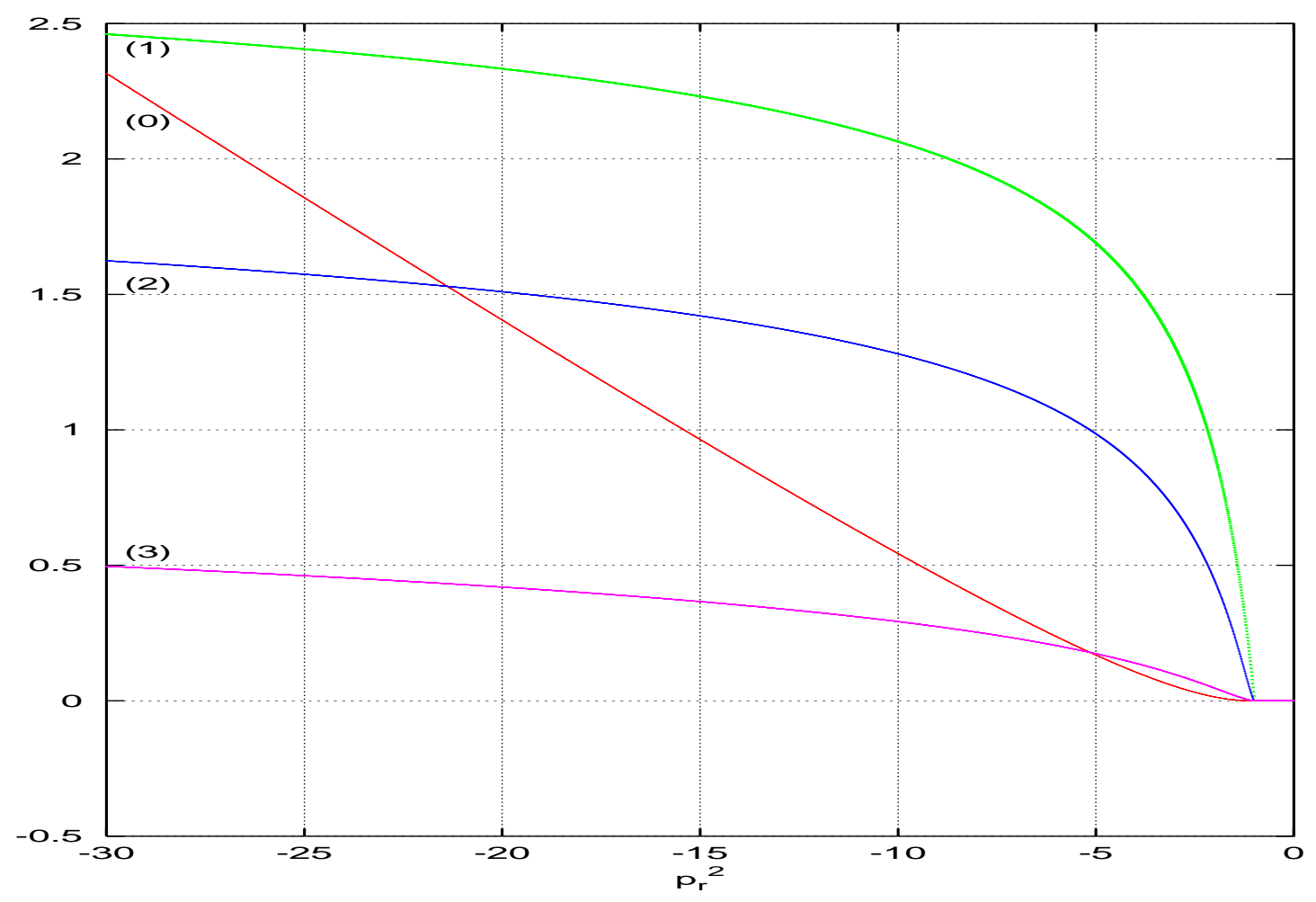

Figure 5: Plots of $\operatorname{Im} F_{0, r}^{(0)}$ (labeled as $\left.(0)\right)$ and $\operatorname{Im} F_{i}^{(0)}$ (labeled as $\left.(i)\right)$ as a function of $p_{r}^{2}$ for $m_{1}=1, m_{2}=9, m_{3}=200$ and $\mu=m_{1}+m_{2}+m_{3}$.

equations simplify, allowing the analytic calculation of the MI; but as also the coefficients of the derivatives vanish there, to start the numerical evaluation from that points the values of the first derivatives must be provided as well. We use for that purpose the analytic values presented in [3, 5, 8]. Starting from $p_{r}^{2}=0$, numerical instabilities arise when approaching any of the pseudo-thresholds; therefore to obtain the value of the MI in the proximity of the threshold $p_{t h, r}^{2}=-1$ or of the pseudo-thresholds $p_{p s 1, r}^{2}, p_{p s 2, r}^{2}, p_{p s 3, r}^{2}$, we take the threshold or pseudo-thresholds themselves as the starting points of the numerical evaluation. Further, for very large values of $p_{r}^{2}$ it is more convenient to start from the value $x=\frac{1}{p_{r}^{2}}=0$, so that the asymptotic expansion at large $p_{r}^{2}$ is also needed.

We use the Runge-Kutta method in the complex plain of $p_{r}^{2}$; the initial condition guarantees as in the real case the uniqueness of the solution, provided that we do not cross the cut [16], which extends in the present case from the threshold $\left(p_{t h, r}^{2}=-1\right)$ to $-\infty$ along the real axis. As already remarked, due to use of Euclidean variables in Eq.(1), 
the proper sign of the imaginary part of the solutions is obtained with a path laying in the lower half complex plane of $p_{r}^{2}$. Using $p_{r}^{2}$ as a complex variable has the advantage that the initial conditions at $p_{r}^{2}=0$ can be used to obtain the solutions everywhere, with a path which does not approach too much pseudo-thresholds and threshold. This feature is relevant, as the method reaches much faster the required accuracy, when starting from $p_{r}^{2}=0$, rather than from anyone of the other special points - even if the result is of course independent of the chosen path.

The program is organized as an independent subroutine, whose arguments are the input values of the masses $m_{i}$, of $p^{2}$ (which is real) and for the required accuracies, and the output values of the MI with their errors. Actually the input accuracies refer separately to the real part, the imaginary part and the absolute value of the functions. However the accuracy of the imaginary part is controlled by the program only when not vanishing, i.e. for $p_{r}^{2}<-1$, but its value is in any case an indication of the precision of the result. The program starts usually from the initial conditions at $p_{r}^{2}=0$. However, if $\left|p_{r}^{2}\right|>900$, it starts from initial conditions at $x \equiv 1 / p_{r}^{2}=0$, while if $\left|p_{r}^{2}-p_{s}^{2}\right|<0.001$, where $p_{s}^{2}$ is the threshold or any of the pseudo-thresholds, it starts from the initial conditions at $p_{s}^{2}$.

When starting from $p_{r}^{2}=0$, the fourth-order Runge-Kutta method is applied to the system of equations Eq.(7) and the required final value of $p_{r}^{2}$ is reached following the rectangular path in the complex plane: $(0,0),(0,-0.1),\left(p_{r}^{2},-0.1\right),\left(p_{r}^{2}, 0\right)$. When pointing to time-like values $p_{r}^{2}<0$, this path has the merit of avoiding the numerically troublesome points of the threshold and pseudo-thresholds. The same path is also used for reaching space-like values $p_{r}^{2}>0$, although no special points occur along on the real axis, as it turns out that the requested precision is usually reached much faster along a complex path than along the real one.

The fourth-order Runge-Kutta for the system of equations Eq.(7) is also used when the starting point of $p_{r}^{2}$ is one of the pseudo-thresholds $p_{p s 1, r}^{2}, p_{p s 2, r}^{2}, p_{p s 3, r}^{2}$, along a similar rectangular path: $\left(p_{p s, r}^{2}, 0\right),\left(p_{p s, r}^{2},-0.1\right),\left(p_{r}^{2},-0.1\right),\left(p_{r}^{2}, 0\right)$.

When starting from $p_{r}^{2}=-1$ (the threshold) the same system of equations cannot be used, as the first derivatives of the master integrals $F_{i}, i=1,2,3$ are infinite at that point. Instead, we introduce new functions $F_{0, r}^{t h}, F_{i}^{t h}$ through the definitions

$$
\begin{aligned}
& F_{0, r}^{(0)}=-\frac{\pi}{32} \sqrt{m_{1, r} m_{2, r} m_{3, r}} x_{t h}^{2} \log \left(x_{t h}\right)+F_{0, r}^{t h} \\
& F_{i}^{(0)}=\frac{\pi}{16} \frac{\sqrt{m_{1, r} m_{2, r} m_{3, r}}}{m_{i, r}} x_{t h} \log \left(x_{t h}\right)+F_{i}^{t h}, i=1,2,3
\end{aligned}
$$

where $x_{t h}=p_{r}^{2}+\left(m_{1, r}+m_{2, r}+m_{3, r}\right)^{2}=p_{r}^{2}+1$, we generate algebraically the system of 
differential equations which they satisfy, and then we solve numerically that new system within the program. We do not report here the new system of equations as one can easily obtain it from the Eq.(7) using Eq.(13). The function $F_{0, r}^{(0)}$ does not have the same problems of the $F_{i}^{(0)}, i=1,2,3$, but the subtraction is performed anyway to simplify the equations. The required value of $p_{r}^{2}$ is then reached along the triangular path in the complex plane $(-1,0),\left(\left(p_{r}^{2}-1\right) / 2,-0.01\right),\left(p_{r}^{2}, 0\right)$.

In the asymptotic region $\left|p_{r}^{2}\right|>900$ we perform the change of variables $p_{r}^{2} \rightarrow x \equiv 1 / p_{r}^{2}$, then we subtract from the MI the terms not vanishing at $x=0$ (the original MI are indeed divergent at $x \rightarrow 0$ ) and finally we write a system of equations for the subtracted MI $F_{0, r}^{a s}$ and $F_{i}^{a s}, i=1,2,3$ defined as

$$
\begin{aligned}
F_{0, r}^{(0)} & =\frac{p_{r}^{2}}{32}\left(\log \left(p_{r}^{2}\right)-\frac{13}{4}\right)+\frac{1}{32} \log ^{2}\left(p_{r}^{2}\right) \sum_{i=1}^{3} m_{i, r}^{2}-\frac{1}{16} \log \left(p_{r}^{2}\right) \sum_{i=1}^{3} m_{i, r}^{2} \log \left(m_{i, r}^{2}\right) \\
& -\frac{1}{32} \sum_{i=1}^{3} m_{i, r}^{2}\left(5-6 \log \left(m_{i, r}^{2}\right)+\log ^{2}\left(m_{i, r}^{2}\right)\right)+F_{0, r}^{a s} \\
F_{i}^{(0)} & =-\frac{1}{32} \log ^{2}\left(p_{r}^{2}\right)+\frac{1}{16} \log \left(p_{r}^{2}\right)\left(\log \left(m_{i, r}^{2}\right)+1\right) \\
& +\frac{1}{32}\left(-1-4 \log \left(m_{i, r}^{2}\right)+\log ^{2}\left(m_{i, r}^{2}\right)\right)+F_{i}^{a s} .
\end{aligned}
$$

Again the new system of equations for $F_{0, r}^{a s}, F_{i}^{a s}$ can be obtained in a simple way substituting Eq.(14) into Eq.(7). The numerical solution is then obtained in the variable $x$ along the complex triangular path $(0,0),(x / 2,-0.01),(x, 0)$.

The errors assigned to the final results of the Runge-Kutta method are estimated by comparing them to the results obtained with a number of steps 10 times smaller then for the final results. The difference of the two results is taken as the estimate of the absolute error. To account for the cumulated rounding error, we estimate the relative error in a $N$ step calculation as $\sqrt{N} \times 10^{-15}$, (as the program works in double precision), and then take the cumulated rounding error as the relative error times the value of the result. We finally take the sum of the absolute error and the cumulated rounding error as an indication of the error in the result.

The initial number of steps $N_{i}$ for $\left|p_{r}^{2}\right|<1$ is taken to be $N_{i}=2 / \min$ (accuracies) (where min(accuracies) is the smallest of the accuracies required in calling the routine) and $N_{i}=2\left|p_{r}^{2}\right| / \min$ (accuracies) for $\left|p_{r}^{2}\right| \geq 1$, but is set to $N_{i}=20$ if the number comes out smaller then 20. If the required precision is not reached the number of steps is increased by a factor 4 , the system is solved once more and the procedure for estimating the error is repeated. For high required accuracy it might happen that the estimated error grows 


\begin{tabular}{cccll}
$p_{r}^{2}$ & $\operatorname{Re} F_{0, r}^{(0)}$ & $\operatorname{Im} F_{0, r}^{(0)}$ & $\operatorname{Re} F_{1}^{(0)}$ & $\operatorname{Im} F_{1}^{(0)}$ \\
\hline$-1000 .-113.349786296(3)$ & $96.922241476(2)$ & $-0.4811355157(1)$ & $3.255217395(1)$ \\
-30. & $-0.230629539580(2)$ & $2.31604333072(1)$ & $2.86686026427(1)$ & $2.46075767262(1)$ \\
-15. & $0.044413679180(8)$ & $0.964565210432(6)$ & $3.47247970401(2)$ & $2.23096593908(2)$ \\
-1.5 & $-0.2536003902785(5)$ & $0.0032295545873(1)$ & $5.03180993338(1)$ & $0.564979990606(8)$ \\
\hline
\end{tabular}

Table 1: The benchmark values of $F_{0, r}^{(0)}$ and $F_{1}^{(0)}$ for masses $m_{1}=1, m_{2}=9, m_{3}=200$ and $\mu=m_{1}+m_{2}+m_{3}$.

\begin{tabular}{rllll}
$p_{r}^{2}$ & $\operatorname{Re} F_{2}^{(0)}$ & $\operatorname{Im} F_{2}^{(0)}$ & $\operatorname{Re} F_{3}^{(0)}$ & $\operatorname{Im} F_{3}^{(0)}$ \\
\hline-1000. & $-1.4689531571(1)$ & $2.393163661(1)$ & $-0.81238274708(3)$ & $1.17931522036(6)$ \\
-30. & $0.880149099634(4)$ & $1.624323639603(8)$ & $0.1097269441157(5)$ & $0.496397637240(4)$ \\
-15. & $1.26943734837(1)$ & $1.42094925579(1)$ & $0.195266696001(4)$ & $0.366097572324(2)$ \\
-1.5 & $2.066516140875(4)$ & $0.239828506599(7)$ & $0.2185459121886(5)$ & $0.0195952353591(3)$ \\
\hline
\end{tabular}

Table 2: The benchmark values of $F_{2}^{(0)}$ and $F_{3}^{(0)}$ for masses $m_{1}=1, m_{2}=9, m_{3}=200$ and $\mu=m_{1}+m_{2}+m_{3}$.

when the number of steps is increased (because of an accumulation of the rounding errors, etc.). In that case the program gives out the best result (i.e. the one with the smallest error). It may also happen, in the case the accuracy obtained in a given step is almost equal to the required one, that in the next step the accuracy obtained is much higher then the required one.

Typical running times on PC with Intel Pentium III $(1 \mathrm{GHz})$ CPU are the following: for required accuracy of $10^{-7}$ a fraction of a second for $\left|p_{r}^{2}\right| \simeq 0.2,2$ seconds for $\left|p_{r}^{2}\right| \simeq 2$ and 8 seconds for $\left|p_{r}^{2}\right| \simeq 30$; for required accuracy of $10^{-11} 20$ seconds for $\left|p_{r}^{2}\right| \simeq 0.2,3.5$ minutes for $\left|p_{r}^{2}\right| \simeq 2$ and 59 minutes for $\left|p_{r}^{2}\right| \simeq 30$.

The program is available from authors upon request and we report in Tables 1.2 and (3) a few results, which can serve as a benchmark. The reported results were all obtained asking the accuracies to be $10^{-11}$. In Table 3 the values at $p_{r}^{2}=0$, at threshold $\left(p_{r}^{2}=-1\right)$ and at three pseudo-thresholds $\left(p_{r}^{2}=p_{p s 1, r}^{2}, p_{p s 2, r}^{2}, p_{p s 3, r}^{2}\right)$ are calculated from the known analytical results [3, 因, 5, 8] incorporated into the program, therefore no error is indicated (the imaginary parts vanish for those values of $p_{r}^{2}$ ). 


\begin{tabular}{cllll}
$p_{r}^{2}$ & $F_{0, r}^{(0)}$ & $F_{1}^{(0)}$ & $F_{2}^{(0)}$ & $F_{3}^{(0)}$ \\
\hline-1 & -0.279454902855371 & 4.83093725524177 & 1.89253423642110 & 0.185424043556224 \\
-0.99 & $-0.2798928396415(5)$ & $4.80520291678(1)$ & $1.884686031133(5)$ & $0.1846128537367(4)$ \\
$p_{p s 3, r}^{2}$ & -0.280281633048667 & 4.78848044412341 & 1.87869903011051 & 0.183940826371101 \\
-0.9 & $-0.2836780811878(5)$ & $4.68669935479(1)$ & $1.836380342548(8)$ & $0.1786103121374(3)$ \\
$p_{p s 2, r}^{2}$ & -0.286233415451605 & 4.62888926299134 & 1.80974413459626 & 0.174898795219002 \\
-0.825 & $-0.2866587055221(5)$ & $4.620058473554(8)$ & $1.805561498336(6)$ & $0.1742958493924(4)$ \\
$p_{p s 1, r}^{2}$ & -0.286906928933491 & 4.61498769104262 & 1.80314761916276 & 0.173945546345814 \\
-0.8 & $-0.2876221116285(5)$ & $4.60069487253(1)$ & $1.796297920597(6)$ & $0.1729424293568(4)$ \\
-0.1 & $-0.3101507246241(3)$ & $4.261426874519(4)$ & $1.619837556072(1)$ & $0.1432933391084(2)$ \\
0 & -0.312816604092084 & 4.22788075922252 & 1.60134292154365 & 0.139821925866842 \\
1.0 & $-0.3340476235037(6)$ & $3.970691522343(6)$ & $1.455289136414(2)$ & $0.1103513887593(2)$ \\
30.0 & $0.323213333716(3)$ & $2.33632892937(2)$ & $0.425519391740(4)$ & $-0.178170159306(2)$ \\
$1000.115 .539777092(3)$ & $-0.8050335305(2)$ & $-1.7888035846(2)$ & $-1.11980894482(3)$ \\
\hline
\end{tabular}

Table 3: The benchmark values of $F_{0, r}^{(0)}, F_{1}^{(0)}, F_{2}^{(0)}$ and $F_{3}^{(0)}$ for masses $m_{1}=1, m_{2}=9$, $m_{3}=200$ and $\mu=m_{1}+m_{2}+m_{3}$.

\section{Tests and comparisons.}

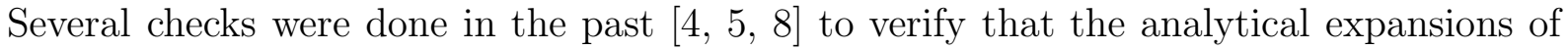
the master integrals in the special points, used within the numerical program, satisfy the differential equations and agree with the results existing in the literature.

A remarkable feature of the extension of the RK-method to the complex plane is that it provides some natural self-consistency checks of the algorithm implementation. Starting from a special point and moving to a chosen value of $p^{2}$ with different paths in the $p^{2}$-complex-plane, the values obtained for the master integrals should agree inside the errors of the method discussed previously. One has however to remember that paths chosen in the upper and lower half-plane, respect to the real axis, give opposite sign to the imaginary part of the master integrals for time-like values of the external invariant above threshold, $p_{r}^{2}<-1$. An even more complete test is to reach the same value of $p^{2}$ starting from different special points, hence following different paths, and compare the values of the master integrals at $p^{2}$ obtained along the various paths. If the values coincide inside the assigned errors the consistency between the differential equations, the expansions in the special points used as initial values, the implementation of the RK-method and the 
algorithm for estimating the errors are cross-tested in a rather effective way.

We have performed several of the mentioned checks in the different regions of $p^{2}$ obtaining the requested agreement.

The only published precise numerical results for the general massive case (all different non-zero mass values) are presented in [2, 11], in the form of a combination of the general massive case with massless cases, to cancel the pole singularities in $(n-4)$. In our notation that combination is

$$
\begin{aligned}
T_{123 N}\left(p^{2}, m_{1}^{2}, m_{2}^{2}, m_{3}^{2}\right)=-16[ & +F_{0}^{(0)}\left(m_{1}^{2}, m_{2}^{2}, m_{3}^{2}, p^{2}\right)-F_{0}^{(0)}\left(m_{1}^{2}, 0, m_{3}^{2}, p^{2}\right) \\
& \left.-F_{0}^{(0)}\left(0, m_{2}^{2}, m_{3}^{2}, p^{2}\right)+F_{0}^{(0)}\left(0,0, m_{3}^{2}, p^{2}\right)\right]
\end{aligned}
$$

which has also the property of being independent of $\mu$; the overall factor $(-16)$ accounts for the different definition of the master integral in Eq.(11) and our $p^{2}$ corresponds to $\left(-p^{2}\right)$ in [2] and to $s$ in [11].

To obtain the values for $F_{0}^{(0)}\left(0,0, m_{3}^{2}, p^{2}\right)$ we use the analytic formula presented in [3], while for the values of $F_{0}^{(0)}\left(m_{1}^{2}, m_{2}^{2}, m_{3}^{2}, p^{2}\right), F_{0}^{(0)}\left(m_{1}^{2}, 0, m_{3}^{2}, p^{2}\right)$ and $F_{0}^{(0)}\left(0, m_{2}^{2}, m_{3}^{2}, p^{2}\right)$ we use the present program. Although the value zero for the masses is not allowed, we have checked that the limit can be in practice reached numerically. Comparing the results obtained for the mass values from $10^{-6}$ to $10^{-9}$, we can estimate the error coming from having a mass not exactly zero, by taking the difference between the results obtained with the two smallest values used for the mass to be set to zero. As the error due to zero mass limit is sometimes comparable with the error due to the RK-method, we sum the two errors for each of the considered functions. The final error of $T_{123 N}$ is the sum of the errors assigned by the algorithm to each of the contributing functions in Eq.(15). The larger errors (or less efficiency in calculations) come from the zero mass contributions, for which an approach based entirely on analytical expressions is in preparation [17]. Furthermore the choice of equal values for two or even all the three masses, reduces the number of the independent equations in the system of differential equations, generating potential numerical problems, although less serious than those for the zero mass.

In [2] the values for $T_{123 N}\left(p^{2}, m_{1}^{2}, m_{2}^{2}, m_{3}^{2}\right)$ are presented, for different sets of the masses $m_{1}, m_{2}, m_{3}$, and for the two regions of small $\left|p^{2}\right|<\left(m_{1}+m_{2}+m_{3}\right)^{2}$ and large $\left|p^{2}\right|>$ $\left(m_{1}+m_{2}+m_{3}\right)^{2}$ in their Table 1 and 2 respectively.

We repeat in Table 1 for the same values of the masses and $p^{2}$ the results of Table 1 of [2] for the multiple series (first entry), pushed to a large number of terms in some cases, and our results (second entry). The results are in excellent agreement.

Also in Table 7 of [11] the values for the same combination $T_{123 N}$ (there called $S_{c}$ ) are 


\begin{tabular}{ccc|cl|rl}
$m_{1}$ & $m_{2}$ & $m_{3}$ & $p^{2}$ & $T_{123 N}$ & $p^{2}$ & $T_{123 N}$ \\
\hline 3 & 3 & 10 & -9 & -7.3129877443 & 9 & -6.93244055931 \\
& & & & $-7.3129877442(26)$ & & $-6.93244055924(50)$ \\
\hline 2 & 3 & 10 & -20 & -4.1493850173 & 20 & -3.63591843327 \\
& & & & $-4.1493850171(18)$ & & $-3.63591843320(95)$ \\
\hline 2 & 2 & 10 & -25 & -2.3353847298 & 25 & -1.9428452190 \\
& & & & $-2.3353847298(14)$ & & $-1.9428452191(10)$ \\
\hline 1 & 2 & 10 & -30 & -0.8117674738 & 30 & -0.6306847352 \\
& & & & $-0.8117674738(15)$ & & $-0.6306847353(12)$ \\
\hline 1 & 1 & 10 & -49 & -0.3167501084 & 49 & -0.1950338472 \\
& & & & $-0.3167501085(24)$ & & $-0.1950338472(21)$ \\
\hline 3 & 4 & 15 & -50 & -7.9471022759 & 50 & -6.8270303849 \\
& & & & $-7.9471022760(33)$ & & $-6.8270303852(19)$ \\
\hline 3 & 4 & 20 & -100 & -6.0171476156 & 100 & -4.9485063889 \\
& & & & $-6.0171476159(59)$ & & $-4.9485063897(42)$ \\
\hline 3 & 4 & 20 & -150 & -6.3903568683 & 150 & -4.7506023184 \\
& & & & $-6.3903568686(73)$ & & $-4.7506023184(66)$ \\
\hline 5 & 5 & 25 & -150 & -14.5339444977 & 150 & -1.21816923644 \\
& & & & $-14.5339444982(87)$ & & $-1.21816923648(68)$ \\
\hline 5 & 5 & 25 & -200 & -15.0523063012 & 200 & -11.8790613597 \\
& & & & $-15.0523063010(95)$ & & $-11.8790613597(83)$ \\
\hline & & & & &
\end{tabular}

Table 4: Comparison for small $\left|p^{2}\right|<\left(m_{1}+m_{2}+m_{3}\right)^{2}$. In each box the first entry is the value of the multiple series of Table 1 of [2], the second entry is our result (the error in the last digits is enclosed in parenthesis). Our value of $p^{2}$ corresponds to $-p^{2}$ in [2]. 


\begin{tabular}{ll|rl|rl}
$p^{2}$ & $T_{123 N}$ & $p^{2}$ & $T_{123 N}$ & $p^{2}$ & $T_{123 N}$ \\
\hline-1 & -70.6856984 & -25 & -70.75620346 & -81 & -70.92141286 \\
& $-70.6856977(39)$ & & $-70.75620352(11)$ & & $-70.92141291(70)$ \\
& -70.686011 & & -70.756299 & & -70.921481 \\
\hline 1 & -70.6798310 & 25 & -70.609519049 & 81 & -70.446146678 \\
& $-70.6798305(21)$ & & $-70.609519051(97)$ & & $-70.446146655(35)$ \\
& -70.680106 & & -70.609231 & & -70.446044 \\
\hline
\end{tabular}

Table 5: Comparison for small $\left|p^{2}\right|<\left(m_{1}+m_{2}+m_{3}\right)^{2}$ and for $m_{1}=10, m_{2}=20, m_{3}=$ 100. In each box the first entry is the multiple series value of [2], the second entry is our result (the error on the last digits is in parenthesis) the third entry is from the numerical integration in Table 7 of [11]. Our value of $p^{2}$ corresponds to $-p^{2}$ in [2] and to $s$ in [11].

presented for small $s$, equal to our $p^{2},\left|p^{2}\right|<\left(m_{1}+m_{2}+m_{3}\right)^{2}$ and for $m_{1}=10, m_{2}=$ $20, m_{3}=100$. They are repeated here in Table 5, where in each box the first entry comes from the multiple series of [2] with a large number of terms, the second entry is the present result, the third entry is from the numerical integration of [11]. Again we have excellent agreement with the multiple series of [2], while the accuracy of the numerical integration of [11] is within a few ppm, inside the relative $10^{-5}$ precision declared there.

In Table 6 we report the results of Table 2 of [2] for the combination $T_{123 N}$, for large and negative $p^{2}$ (i.e. $\left|p^{2}\right|>\left(m_{1}+m_{2}+m_{3}\right)^{2}$, the value of $p^{2}$ here corresponds to $-p^{2}$ in [2]), so that this time there is also an imaginary part. In each box the first entry comes from the multiple series of [2], with a large number of terms, the second entry is the present result. Again we have excellent agreement with the multiple series of [2] in most of the cases, in few cases there is a deviation of two times the assigned error, that we attribute to our procedure of approaching the zero mass. In the seventh box we assign an error also to the multiple series, because, although each sum is taken up to 70 terms, the results are not yet stable. The assigned error is the difference with the sums taken up to 60 terms. We attribute the difficulty to the chosen value of $p^{2}=-150$, which is too near to the threshold value $-(3+4+5)^{2}=-144$.

In Table 7 we report the results of Table 2 of [2] for the combination $T_{123 N}$, for large and positive $p^{2}$ (i.e. $\left|p^{2}\right|>\left(m_{1}+m_{2}+m_{3}\right)^{2}$; our $p^{2}$ corresponds to $-p^{2}$ in [2]). In each box the first entry comes from the multiple series of [2], with a large number of terms, the second entry is the present result. Also here we have excellent agreement with the multiple series of [2] in most of the cases, in few cases there is a deviation about two times the assigned error, that we attribute to our procedure of approaching the zero mass. 


\begin{tabular}{crrr|ll}
$m_{1}$ & $m_{2}$ & $m_{3}$ & $p^{2}$ & $\operatorname{Re} T_{123 N}$ & $\operatorname{Im~} T_{123 N}$ \\
\hline 2 & 3 & 2 & -80 & 0.587432001 & -11.262835755 \\
& & & & $0.587431990(41)$ & $-11.262835744(24)$ \\
\hline 3 & 3 & 3 & -100 & -1.28284949 & -20.899600723 \\
& & & & $-1.28284943(16)$ & $-20.899600741(10)$ \\
\hline 2 & 3 & 4 & -100 & -0.3286481685 & -11.84587606309 \\
& & & & $-0.3286481687(16)$ & $-11.84587606302(60)$ \\
\hline 3 & 4 & 4 & -150 & -1.26795173 & -26.491194705 \\
& & & & $-1.26795173(21)$ & $-26.491194694(41)$ \\
\hline 2 & 4 & 3 & -150 & 1.5662482672 & -11.9689438774 \\
& & & & $1.5662482670(14)$ & $-11.9689438778(12)$ \\
\hline 3 & 3 & 4 & -150 & 0.9865824304 & -16.10213970663 \\
& & & & $0.9865824312(19)$ & $-16.10213970687(77)$ \\
\hline 3 & 4 & 5 & -150 & $-4.7638745(12)$ & $-29.601246304(26)$ \\
& & & & $-4.7638748416(32)$ & $-29.60124631204(68)$ \\
\hline 2 & 3 & 4 & -200 & 1.6960823345 & -6.02417248918 \\
& & & & $1.6960823350(17)$ & $-6.02417248906(81)$ \\
\hline 3 & 4 & 4 & -200 & 1.86355967 & -20.39852237 \\
& & & & $1.86355979(34)$ & $-20.39852240(12)$ \\
\hline 4 & 4 & 4 & -250 & 2.64395201 & -27.60904430 \\
& & & & $2.64395222(31)$ & $-27.60904439(18)$ \\
\hline & & & & &
\end{tabular}

Table 6: Comparison for $p^{2}$ large $\left(\left|p^{2}\right|>\left(m_{1}+m_{2}+m_{3}\right)^{2}\right)$ and negative. In each box first entry is the multiple series value of Table 2 of [2], the second entry is our result (the error on the last digits is in parenthesis). Our $p^{2}$ corresponds to $-p^{2}$ in [2].

\begin{tabular}{rrr|rl|rrr|rl}
$m_{1}$ & $m_{2}$ & $m_{3}$ & $p^{2}$ & $T_{123 N}$ & $m_{1}$ & $m_{2}$ & $m_{3}$ & $p^{2}$ & $T_{123 N}$ \\
\hline 2 & 2 & 2 & 50 & -3.728125558 & 2 & 4 & 3 & 100 & -7.1836810855 \\
& & & $-3.728125610(46)$ & & & & & $-7.1836810854(54)$ \\
\hline 3 & 3 & 3 & 100 & -8.79126989 & 3 & 4 & 3 & 120 & -12.430997190 \\
& & & $-8.79127000(11)$ & & & & & $-12.430997250(30)$ \\
\hline 3 & 3 & 4 & 150 & -7.0830520665 & 3 & 4 & 4 & 150 & -10.85647158 \\
& & & & $-7.0830520661(28)$ & & & & & $-10.85647158(26)$ \\
\hline 3 & 4 & 3 & 150 & -11.361931056 & 3 & 4 & 4 & 200 & -9.64611359 \\
& & & & $-11.361931121(31)$ & & & & & $-9.64611360(29)$ \\
\hline 2 & 3 & 4 & 200 & -3.3018636831 & 4 & 4 & 4 & 250 & -13.57188440 \\
& & & & $-3.3018636830(21)$ & & & & & $-13.57188463(21)$ \\
\hline
\end{tabular}

Table 7: Comparison for $p^{2}$ large $\left(\left|p^{2}\right|>\left(m_{1}+m_{2}+m_{3}\right)^{2}\right)$ and positive. In each box first entry is the multiple series value of Table 2 of [2], the second entry is our result (the error on the last digits is in parenthesis). Our $p^{2}$ corresponds to $-p^{2}$ in [2]. 


\section{Conclusions.}

We propose to solve numerically, by means of the Runge-Kutta method extended to the complex plane, the system of the differential equations satisfied by the MI related of the diagrams, which due to the large number of occurring parameters cannot be calculated analytically.

We apply the method to the study of the simplest non trivial diagram, the general massive 2-loop sunrise self-mass, which is already exhibiting a number of intriguing analytic properties. We obtain, for all the allowed values of the parameters and of the external invariant $p^{2}$, very accurate values of the MI within reasonable CPU time, in good agreement with the results already present in the literature.

The method can be naturally extended to the other self-mass diagrams of the same order, like the 2-loop 4-propagator self-mass diagram for which the differential equation is already known [18].

The extension to higher order self-mass diagrams will only increase linearly the number of the MI and of the differential equations in the system, while the growing of the number of parameters is not a problem at all. Also the extension to diagrams with three or more external legs, which means multi variable cases, can be easily envisaged.

The true difficulty of the method is the need of initial conditions for starting the numerical solution of the differential equations; clearly the initial conditions have to be provided by an independent method. Of special values are, in this respect, the special points (such as $0, \infty$, thresholds and pseudothresholds) where an analytic calculation is easier and sometimes possible, as in the case discussed in this paper. When the special points are used, also the first derivative of the MI have to be provided as an independent input to the Runge-Kutta approach, but that is analytically a relatively simpler task, amounting to an iteration of the expansions provided by the differential equations.

Acknowledgments. We thank Sandro Rambaldi for his invaluable advise on the use of Runge-Kutta method to solve differential equations.

One of us (HC) is grateful to the Bologna Section of INFN and to the Department of Physics of the Bologna University for support and kind hospitality. 


\section{A Corrections to some analytic formulae of [5, 8$]$}

We report here for completeness the correct form of the formulae, which are wrongly reported in our previous publications [5, 8] and are used here to obtain the numerical values of the MI at pseudo-thresholds and threshold.

In section 5 of [5] there are three misprints. In Eq.(41) the factor $\frac{1}{16}$ in front of the integral should be missing. In the first line of Eq.(44) $\log (y) \operatorname{should} \operatorname{read} \log \left(y_{S}\right)$ and in Eq.(47) there is a missing factor 4 in front of $\mathcal{I}_{2}$.

In [8] there is one misprint in Eq.(45): the second line from the end should be of the opposite sign $\left({ }^{\prime}-^{\prime} \rightarrow^{\prime}{ }^{\prime}\right)$. In Eq.(31) of [8] the expression for $\mathcal{I}_{3}\left(m_{1}, m_{2}, m_{3}\right)$ should be symmetric in all the masses, so it becomes

$$
\mathcal{I}_{3}\left(m_{1}, m_{2}, m_{3}\right)=\tilde{\mathcal{I}}_{3}\left(m_{1}, m_{2}, m_{3}\right)+\tilde{\mathcal{I}}_{3}\left(m_{1}, m_{1}, m_{2}\right)-\tilde{\mathcal{I}}_{3}\left(m_{2}, m_{1}, m_{1}\right)
$$

with

$$
\begin{aligned}
& \quad \tilde{\mathcal{I}}_{3}\left(m_{1}, m_{2}, m_{3}\right)=\sqrt{m_{1} m_{2}} \int d m_{3} \frac{1}{\sqrt{\left.m_{3} m_{1}+m_{2}+m_{3}\right)}}\left[\frac{\log \left(\frac{m_{3}}{m_{1}}\right)}{m_{3}+m_{1}}+\frac{\log \left(\frac{m_{3}}{m_{2}}\right)}{m_{3}+m_{2}}\right]= \\
& i\left\{\log \left(\frac{m_{1}+m_{2}}{4 m_{1}}\right)\left[\log \left(t-t_{1}\right)-\log \left(t-t_{2}\right)\right]+\log \left(\frac{m_{1}+m_{2}}{4 m_{2}}\right)\left[\log \left(t+t_{2}\right)-\log \left(t+t_{1}\right)\right]\right. \\
& \quad+\log \left(t-t_{1}\right)\left[2 \log \left(1-t_{1}\right)-\log \left(t_{1}\right)\right]-\log \left(t-t_{2}\right)\left[2 \log \left(1-t_{2}\right)-\log \left(t_{2}\right)\right] \\
& \quad-\log \left(t+t_{1}\right)\left[2 \log \left(1+t_{1}\right)-\log \left(-t_{1}\right)\right]+\log \left(t+t_{2}\right)\left[2 \log \left(1+t_{2}\right)-\log \left(-t_{2}\right)\right] \\
& \quad-2 \operatorname{Li}_{2}\left(\frac{t-t_{1}}{1-t_{1}}\right)+2 \operatorname{Li}_{2}\left(\frac{t-t_{2}}{1-t_{2}}\right)+\operatorname{Li}_{2}\left(-\frac{t-t_{1}}{t_{1}}\right)-\operatorname{Li}_{2}\left(-\frac{t-t_{2}}{t_{2}}\right) \\
& \left.\quad+2 \operatorname{Li}_{2}\left(\frac{t+t_{1}}{1+t_{1}}\right)-2 \operatorname{Li}_{2}\left(\frac{t+t_{2}}{1+t_{2}}\right)-\operatorname{Li}_{2}\left(\frac{t+t_{1}}{t_{1}}\right)+\operatorname{Li}_{2}\left(\frac{t+t_{2}}{t_{2}}\right)\right\}
\end{aligned}
$$

where the expressions of the logarithms account now properly for their imaginary part. Consequently the Eq.(42) of [8] should be replaced by

$$
b-\frac{K}{32}=\pi\left(-\frac{1}{32}+\frac{5}{32} \log (2)\right)+\frac{1}{8} \mathrm{Cl}_{2}\left(\frac{\pi}{2}\right) .
$$




\section{References}

[1] F.V. Tkachov, Phys. Lett.B 100, 65 (1981); K.G. Chetyrkin and F.V. Tkachov, Nucl. Phys.B 192, 159 (1981).

[2] F.A. Berends, M. Böhm, M. Buza and R. Scharf, Z. Phys.C 63, 227 (1994).

[3] M. Caffo, H. Czyż, S. Laporta and E. Remiddi, Nuovo Cim.A 111, 365 (1998), hep-th/9805118.

[4] F.A. Berends, A.I.Davydychev, N.I. Ussyukina, Phys. Lett.B 426, 95 (1998), hepph/9712209.

[5] M. Caffo, H. Czyż and E. Remiddi, Nucl. Phys.B 581, 274, (2000), hep-ph/9912501, see also the Appendix of the present article.

[6] A.I. Davydychev and V.A. Smirnov, Nucl. Phys.B 554, 391 (1999), hep-ph/9903328.

[7] S. Groote and A.A. Pivovarov, Nucl. Phys. B 580, 459 (2000), hep-ph/0003115.

[8] M. Caffo, H. Czyż and E. Remiddi, Nucl. Phys.B 611, 503, (2001), hep-ph/0103014, see also the Appendix of the present article.

[9] P. Post and J.B. Tausk, Mod. Phys. Lett.A 11, 2115 (1996), hep-ph/9604270.

[10] S. Groote, J.G. Körner and A.A. Pivovarov, Eur. Phys. J.C 11, 279 (1999), hepph/9903412; Nucl. Phys.B 542, 515 (1999), hep-ph/9806402.

[11] G. Passarino, Nucl. Phys.B (2001), hep-ph/0108252.

[12] S. Laporta, Int. J. Mod. Phys.A 15, 5087 (2000), hep-ph/0102033; Phys. Lett.B 504, 188 (2001), hep-ph/0102032.

[13] O.V. Tarasov, Nucl. Phys.B 502, 455 (1997), hep-ph/9703319.

[14] W.H. Press, S.A. Teukolsky, W.T. Vetterling, B.P. Flannery Numerical Recipes in FORTRAN. The Art of Scientific Computing., Cambridge University Press, 1994.

[15] C. Itzykson and J.B. Zuber, Quantum Field Theory, McGraw-Hill, New York, 1980, Sect. 6-3 pag. 301, and references therein.

[16] E.L. Ince, Ordinary differential equations, Dover Publications, New York, 1956. 
[17] H. Czyż, A. Grzelinska and M. Zabawa, in preparation.

[18] M. Caffo, H. Czyż, S. Laporta and E. Remiddi, Acta Phys. Pol.B 29, 2627 (1998), hep-th/9807119. 